\title{
Ciclo ovárico y jerarquía folicular de Peltocephalus dumerilianus (Testudines: Podocnemididae)
}

\author{
Jaime De La Ossa VELASQUEZ1 ${ }^{1}$ Richard Carl VOGT²
}

\section{RESUMÉN}

Se analizaron cuarenta pares de ovarios de hembras adultas de Peltocephalus dumerilianus (Schweigger 1812), para establecer los cambios morfológicos mensuales producidos durante un año. Se llevaron a cabo exámenes macroscópicos de los oviductos y de los ovarios de especímenes que los habitantes ribereńos del municipio de Barcelos, Amazonas, Brasil, utilizaron para su consumo, usando metodológicamente la medida del diámetro y conteo de los folículos ováricos y de los cuerpos lúteos. Los resultados muestran la presencia gradual de folículos acorde con la época reproductiva, resaltando que durante el último tercio del proceso, justo antes de la postura se hacen más evidentes; se analiza la relación existente entre el tamaño de la hembra y la masa ovárica, la cual es estadísticamente significativa $(\mathrm{F}(10,29)=3,655, \mathrm{p}<0,05)$. Se hace evidente la existencia de una jerarquía ovario folicular que demuestra que para esta especie solo es posible fisiológicamente una postura por año.

PALABRAS-CLAVE: Ovarios, folículos, ciclo reproductivo, Peltocephalus dumerilianus, Brasil.

\section{Ovarian cycle and follicular hierarchy of Peltocephalus dumerilianus (Testudines: Podocnemididae)}

\section{ABSTRACT}

Forty pairs of ovaries of mature female Peltocephalus dumerilianus (Schweigger 1812) were analyzed, to establish the morphologic monthly changes that take place during one year; we examined macroscopically the oviducts and the ovaries of specimens that the riverside inhabitants of the municipality of Barcelos, Amazons, Brazil, used for their consumption. We measured the diameter and recorded the number of enlarged ovarian follicles and corpora lutea. The results show the gradual enlargement of follicles in agreement with the reproductive period, observing that during the last third of the process; just before oviposition they become more evident. The positive relationship between the size of the female and the ovarian mass, is statistically significant $(\mathrm{F}(10.29)=3.655, \mathrm{p}<0.05)$. Follicullar sizes suggest that this species nests only once a year.

KEYWORDS: Ovaries, follicles, reproductive cycle, Peltocephalus dumerilianus, Brazil.

1 Universidad de Sucre. jaimedelaossa@yahoo.com

2 Instituto Nacional de Pesquisas da Amazônia. vogt@inpa.gov.br 


\section{INTRODUCCIÓN}

De las especies de Podocnemididae existentes en la Amazonía brasileira se tiene información sobre biología reproductiva de Podocnemis expansa (Vanzolini 1977; Vanzolini y Gómes 1979; Valenzuela 2001), Podocnemis unifilis (Souza y Vogt 1994), Podocnemis sextuberculata (Pezzuti 1998; Bernhard 2001) y para Peltocephalus dumerilianus (Vogt et al. 1994; Vogt 2001; Felix-Silva 2004); pero no se registran estudios sobre ciclo ovárico para estas especies de quelonios.

De manera particular Girlin (2002) menciona que el oviducto de los reptiles es un órgano complejo con una variedad de funciones que incluyen: producción de albumina, producción de membranas del huevo, ovipostura y almacenamiento de esperma. Esas funciones están controladas por elaborados mecanismos fisiológicos que todavía no están totalmente entendidos.

Por su parte Etches y Petitte (1990) proponen la existencia de una jerarquía ovario-folicular como característica de aves y reptiles, que se manifiesta por la presencia ovárica de folículos en todos los estados de maduración. En los reptiles, la jerarquía ovario-folicular incluye los folículos no vitelogénicos y en algunas especies incluye los folículos destinados a ser atrésicos como estructuras de producción hormonal, las dos últimas características diferencian a los reptiles de las aves.

Clark et al. (2001) conceptúan que existe previa diferencia en el tamaño de los folículos que se producen y se liberan en el oviducto, lo cual se relacionaría con el tamaño de los huevos y el tamaño de la nidada. Igualmente se plantea que existe una relación directa entre el tamaño de la hembra y el tamaño de la nidada (Smith y Fretwell 1974; Congdon y Gibbons 1987), también se puede observar diferencialmente que los oviductos en algunas especies de quelonios llegan a contener diferentes tamaños de huevos (Tucker y Janzen 1998).

En cuanto a la influencia que poseen los factores ambientales sobre la reproducción de los quelonios acuáticos continentales, se puede establecer que para la región amazónica, el ciclo reproductivo está condicionado de forma representativa por las variaciones que presentan los flujos de los ríos (Vanzolini 2003), lo cual es denominado elasticidad que es el cociente entre la superficie ocupada durante la fase de máximo anegamiento y/o inundación, y la que corresponde al momento de sequía extrema (Neiff et al. 1994), en la cual interactúan intercaladamente los denominados pulsos del río (Sioli 1975).

En el presente estudio por primera vez se analiza el ciclo ovárico de $P$. dumerilianus y se determina si existe una la relación entre tamaño de la hembra y masa ovárica. Como esta especies es capturado parar comer intensivamente en muchos lugares, esta estudio puede apoyar planes de manejo esta especies determinando su potencial reproductivo por año por tamaño de hembra. Diferente de los especies de Podocnemis, Peltocephalus populaciones son mas resistente de cazadores, por que ellas no hacer sus nidos juntos en playas, sus nidos son solitarios entra la selva, entre dos o tres $m$ de igarapés. En esta manera no esta fácil para exterminar una populación de Peltocephalus y ellos puede sobrevivir con extracción limitado.

\section{MATERIALE Y MÉTODOS}

\section{Amostras}

Las muestras biológicas para el presente trabajo fueron colectadas en la comunidad de São Luís, municipio de

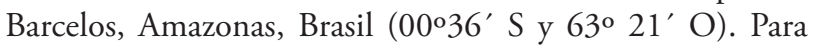
el reconocimiento del ciclo ovárico de la población en estudio se analizaron 40 tractos reproductivos de hembras adultos (ovarios y oviductos), entre dos y cinco tractos reproductivos por mes durante un año (2005-2006), este material era proveniente de las capturas de $P$. dumerilianus que los habitantes locales utilizaron como caza de subsistencia para su consumo; las capturas provenían del medio río Itú, caracterizado como un típico hábitat de igapó (Schôngart et al. 2005).

\section{Procesamiento}

La conservación del material fue realizada durante cuatro días en una solución de formol al 10\%, tamponado con $4 \mathrm{~g} \mathrm{~L}^{-1}$ de $\mathrm{H}_{2} \mathrm{NaPO}_{4} \mathrm{H}_{2} \mathrm{O}$ y $6,05 \mathrm{~g} \mathrm{~L}^{-1}$ de $\mathrm{HNa}_{2} \mathrm{PO}_{4} \mathrm{H}_{2} \mathrm{O}$, después se fijaron en alcohol al 75\%; tres días antes de los análisis de laboratorio, fueron hidratadas con agua destilada.

\section{Avaliación}

Largo total carapax (LTC) fueron tomadas en un línea recto mediante un calibrador digital era medida en $\mathrm{mm}$. Los exámenes macroscópicos de los oviductos y de los ovarios se condujeron para determinar madurez y estado de desarrollo ovárico, usándose el método de medida del diámetro y conteo de los folículos ováricos y de los cuerpos lúteos según la metodología de Ramo (1982), la cual establece las siguientes divisiones: clase I (menor $5 \mathrm{~mm}$ ); clase II ( 5 a $15 \mathrm{~mm}$ ); clase III (mayor a $15 \mathrm{~mm}$ ) y clase IV (con cuerpos lúteos); las medidas fueron tomadas mediante un calibrador digital de 0 - 200 $\mathrm{mm}$ (Mitutoyo ${ }^{\circ} \mathrm{IP} 66 \pm 0,01 \mathrm{~mm}$ ) y las observaciones se realizaron con microscopio binocular (Leica ${ }^{\circledR}$ Zoom 10 x 4); los datos fueron correlacionados con los meses de año, según Avendaño et al. (2002).

\section{Analicé estadística}

Para la morfometria ovárica, presencia de cuerpos lúteos y clases de los ovocitos, respeto al tiempo, se aplicó ANOVA. El número de huevos oviductales por oviducto, así como el número de cuerpos lúteos fueron estadísticamente analizados mediante la prueba de $X^{2}$ (Zar 1996). Ambientalmente 
Tabla 1 - Morfometria del sistema gonadal femenino de P. dumerilianus. (LTC largo total carapax, WOD Peso ovario derecho, WOI peso ovario izquierdo, DoviD diámetro oviducto derecho, Dovil diámetro oviducto izquierdo, DMovoD diámetro máximo ovocitos derechos, DMovol diámetro máximo ovocitos izquierdos, CLD cuerpos lúteos derechos, CLI cuerpos lúteos izquierdos, HOVID huevos oviductales derechos, HOVI huevos oviductales izquierdos)

\begin{tabular}{|c|c|c|c|c|c|c|c|c|c|c|c|}
\hline Mes & LTC & WOD & WOI & DOviD & DOvil & DMOvoD & DMOvol & CLD & CLI & HOVID & HOVII \\
\hline 1 & 26,3 & 2,043 & 4,403 & 3,9 & 3,85 & 2,9 & 3,1 & & & & \\
\hline 1 & 28,4 & 3,35 & 2,49 & 2,3 & 2,35 & 1,98 & 1,94 & & & & \\
\hline 1 & 25,2 & 1,31 & 1,21 & 2,1 & 2,15 & 1,15 & 1,18 & & & & \\
\hline 2 & 27,1 & 1,64 & 1,68 & 1,93 & 1,84 & 1,98 & 2,01 & & & & \\
\hline 2 & 28,4 & 2,44 & 2,5 & 1,78 & 1,88 & 2,2 & 2,15 & & & & \\
\hline 2 & 27,3 & 2,28 & 1,83 & 1,26 & 1,2 & 1 & 1 & & & & \\
\hline 3 & 28,2 & 2,34 & 2,41 & 2,2 & 2,2 & 3,1 & 2,9 & & & & \\
\hline 3 & 27,2 & 2,47 & 2,42 & 4,81 & 4,64 & 2,85 & 2,68 & & & & \\
\hline 3 & 29,6 & 3,74 & 3,47 & 3,2 & 3,3 & 1 & 1 & & & & \\
\hline 4 & 32,4 & 6,61 & 7,15 & 5,94 & 5,93 & 3,86 & 3,11 & & & & \\
\hline 4 & 31,4 & 4,2 & 4,28 & 3,9 & 4,1 & 2,2 & 2,6 & & & & \\
\hline 4 & 34,2 & 5,5 & 5,4 & 4,6 & 4,8 & 2,6 & 3,8 & & & & \\
\hline 5 & 31,5 & 5,5 & 5,4 & 6,3 & 5,8 & 3,8 & 3,9 & & & & \\
\hline 5 & 33,9 & 5,8 & 5,9 & 5,1 & 4,6 & 4,1 & 3,9 & & & & \\
\hline 5 & 33,1 & 7,2 & 7,3 & 4,9 & 5,2 & 2,6 & 2,9 & & & & \\
\hline 6 & 31,5 & 9,45 & 10,2 & 5,25 & 5,22 & 5,8 & 5,6 & & & & \\
\hline 6 & 29,8 & 4,93 & 4,69 & 6,47 & 6,48 & 3,63 & 3,89 & & & & \\
\hline 7 & 35,5 & 23,91 & 22,7 & 6,8 & 6,9 & 13,8 & 14,5 & & & & \\
\hline 7 & 31,4 & 13,89 & 17,05 & 9,3 & 9,4 & 6,73 & 7,95 & & & & \\
\hline 7 & 27,8 & 6,46 & 3,66 & 9,4 & 10,2 & 4,3 & 4,43 & & & & \\
\hline 8 & 35,8 & 35,43 & 34,3 & 12,2 & 11,9 & 24,6 & 21,6 & & & & \\
\hline 8 & 30,5 & 18,56 & 9,574 & 17,45 & 14,9 & 16,8 & 10,11 & & & & \\
\hline 8 & 32,4 & 23,33 & 28,4 & 18,5 & 17,9 & 4,07 & 5,1 & & & & \\
\hline 8 & 33,4 & 15,22 & 18,15 & 12,4 & 12,6 & 8,36 & 5,17 & & & & \\
\hline 9 & 25,2 & 3,75 & 3,69 & 6,73 & 6,81 & 2,56 & 2,63 & & & & \\
\hline 9 & 27,4 & 10,93 & 9,39 & 18,62 & 21,3 & 14,24 & 9,68 & & & & \\
\hline 9 & 31,1 & 12,57 & 12,26 & 17,07 & 15,9 & 12,17 & 14,49 & 4 & 4 & 4 & 5 \\
\hline 9 & 35,2 & 33,15 & 32,94 & 24,16 & 24,2 & 19,81 & 19,15 & 6 & 5 & 6 & 6 \\
\hline 10 & 34,2 & 20,24 & 23,87 & 11,59 & 16,8 & 17,59 & 15,88 & 8 & 6 & 8 & 6 \\
\hline 10 & 33,3 & 24,92 & 25,13 & 21,67 & 19,4 & 7,14 & 6,98 & 6 & 5 & 7 & 6 \\
\hline 10 & 34,2 & 48,25 & 45,66 & 22,94 & 23,5 & 23,37 & 24,68 & 7 & 6 & 7 & 7 \\
\hline 10 & 31,5 & 15,25 & 19,7 & 28,1 & 29,5 & 3,48 & 4,88 & 4 & 2 & 4 & 5 \\
\hline 11 & 35,4 & 29,08 & 23,4 & 21,8 & 19,8 & 9,91 & 11,61 & 6 & 3 & 7 & 6 \\
\hline 11 & 35,2 & 10,6 & 15,4 & 20,42 & 20,6 & 4,3 & 4,57 & 3 & 7 & 5 & 7 \\
\hline 11 & 31,2 & 21,3 & 22,2 & 22,4 & 22,5 & 10,15 & 6,96 & 4 & 5 & 5 & 5 \\
\hline 11 & 30,2 & 4,52 & 4,48 & 3,92 & 3,96 & 1,65 & 1,86 & & & & \\
\hline 11 & 28,9 & 3,65 & 3,68 & 3,22 & 3,41 & 2,05 & 2,14 & & & & \\
\hline 12 & 28,5 & 2,44 & 2,52 & 3,45 & 3,38 & 2,31 & 2,34 & & & & \\
\hline 12 & 31,2 & 1,35 & 1,34 & 1,69 & 1,73 & 1,3 & 1,32 & & & & \\
\hline 12 & 30,4 & 1,77 & 1,78 & 2,68 & 2,65 & 1,25 & 1,19 & & & & \\
\hline
\end{tabular}


se contó con la autorización IBAMA/RAN (0129/2005 y 059/2006).

\section{RESULTADOS}

Se analizaron 40 pares de ovarios y los oviductos correspondientes de hembras maduras (Tabla 1). Al comparar mediante ANOVA la morfometria de las gónadas por mes, todas las variables dependientes analizadas fueron estadísticamente significativas: peso de ovarios, diámetro de los ovocitos, diámetro del oviducto, cuerpos lúteos totales, diámetro del cuerpo lúteo y de los huevos oviductales (F (66, $128,5)=1,667, \mathrm{p}<0,05)$. Por otro lado, al aplicar ANOVA se halló valor significativo para la relación largo total del carapax en línea recta (LC) al compararlo con el peso de los ovarios $(\mathrm{F}(10,29)=3,655, \mathrm{p}<0,05)$.

De forma general y de acuerdo con el incremento morfométrico ovárico y oviductal en función del tiempo, se determinaron cuatro estadios: durante los cinco primeros meses del año cuando el sistema reproductivo está en reposo (aquiescente); después, durante los meses junio a julio, comienza un crecimiento paulatino y activo que en los tres meses siguientes alcanza su mayor expresión, coincidiendo con la época reproductiva; y finaliza con una fase de recuperación en los dos últimos meses del año. Al aplicar ANOVA se obtiene valor significativo para el aumento periódico de los folículos $(\mathrm{F}(11,28)=2,649, \mathrm{p}<0,05)$.

Los cuatro estadios de desarrollo gonadal están asociados a cuatro clases de diámetro folicular: clase I (menor $5 \mathrm{~mm}$ ); clase II ( 5 a $15 \mathrm{~mm}$ ); clase III (mayor a $15 \mathrm{~mm}$ ) y clase IV (presencia de cuerpo lúteo), que se manifiestan significativamente durante el ciclo anual: ANOVA $(F(11,82)=2,779, \mathrm{p}<0,05)$. Igualmente se determina gráficamente la existencia de una jerarquía gonadal conforme a la frecuencia de ocurrencia de

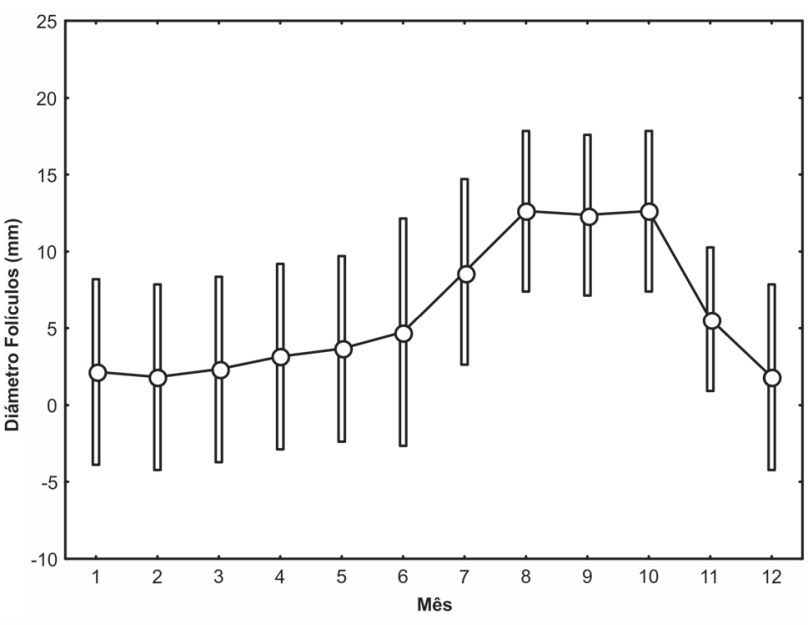

Figura 1 - Crecimiento folicular anual para Peltocephalus dumerilianus, se señalan los meses de mayor incremento en tamaño $(\mathrm{mm})(\mathrm{N}=40)$ las tres clases de folículos, que se hace evidente desde julio hasta noviembre (Figura 1). No existe diferencia significativa entre el número de huevos presentes en cada uno de los dos oviductos $\left(\mathrm{X}^{2}=1,804\right.$, GL $\left.8, \mathrm{p}=0,986\right)$; tampoco existe diferencia significativa entre el número de cuerpos lúteos y huevos oviductales $\left(\mathrm{X}^{2}=7,8759\right.$, GL 8, $\left.\mathrm{p}=0,445\right)$.

\section{Discusión}

Existe la presencia de folículos de todos los tamaños, pertenecientes a las clases I a III, durante el período comprendido entre el séptimo y décimo primer mes del año, presentándose como la época de mayor complejidad ovárica, abarcando desde el inicio del período de maduración ovárica hasta el momento de la postura, pudiendo inclusive, durante ese lapso existir la presencia de huevos oviductales y cuerpos lúteos consecuentemente, queda claro que se establece una jerarquía ovario-folicular que, como lo registran Etches y Petitte (1990), lo cual es una característica de aves y reptiles, que está presente en esta especie de quelonio. Igualmente, por los resultados analizados, teniendo como base el desarrollo folicular, se puede afirmar que $P$. dumerilianus posee solamente una postura anual, ligada a los ciclos hidrológicos de su hábitat o los denominados pulsos del Río y específicamente en la época de sequía (Sioli 1975; Neiff et al. 1994; Vanzolini 2003).

Coincidiendo con Mitchell (1985), Moll y Legler (1971), se determinan para $P$. dumerilianus en este estudio cuatro clases de tamaños de ovocitos que se relacionan con la época del ańo y con la actividad ovárica; igualmente Mahmoud y Kicka (1979) establecen cuatro clases de tamańo, en las cuales la característica tenida en cuenta es el grado de crecimiento folicular: crecimiento mínimo, crecimiento máximo, crecimiento medio y ausencia de crecimiento, las cuales se ajustan a lo observado en el presente trabajo; por los resultados, se acoge lo establecido por Ramo (1982) para Podocnemis vogli, en cuanto a los intervalos de medidas y clases establecidas: menores de cinco mm, entre cinco e 15 $\mathrm{mm}$, mayores de $15 \mathrm{~mm}$, y con cuerpo lúteo, ya que se logra adecuada semejanza en el desarrollo de los ovocitos para ambas especies de Podocnemididae.

La jerarquía ovario-folicular se manifiesta por la presencia ovárica de folículos en todos los estados de maduración, incluyendo en está jerarquía los folículos no vitelogénicos, que es una característica propia de los reptiles (Etches y Petitte 1990). Se puede asumir la jerarquía como una estrategia reproductiva que permite mantener disponibles el mayor número posible de folículos, reduciendo las probables perdidas por excesiva y efectiva ovulación, posibilitando la rápida recuperación ovárica, lo que garantizaría una reproducción máxima de las hembras en cada estación con menor inversión energética. Tales características pueden incrementarse en función del tamaño de la hembra, como sucede en quelonios, ya que existe relación significativa entre el tamaño de la hembra 
y el tamańo de la nidada como lo seńalan: Daza y Paez (2007) para Trachemys callirostris callirostris, Iverson y Smith (1993) para Chrysemys picta, Medem (1983) para P. dumerilianus, Nieuwolt-Dacanay (1997) para Terrapene ornata luteola, Rowe (1994) para Chrysemys picta bellii, y Zuffi et al. (1999) para Emys orbicularis.

En el presente estudio la relación entre el tamaño de la hembra y el peso ovárico fue significativa, indicando un mayor crecimiento gonadal, que representa una mayor masa folicular desarrollada y sustenta la relación del tamaño de la nidada y el tamańo de la hembra positivamente, conforme lo exponen para diversas especies de quelonios Brooks et al. (1992); Congdon y Gibbons (1985); Gibbons et al. (1982). Claramente se observa en este estudio que el potencial reproductivo está en relación directa con la masa corporal de la hembra. Se acepta que la masa corporal de la hembra, en ese caso, se relaciona con la calidad y cantidad de hábitat disponible y aprovechable, así como con factores genéticos individuales y calidad de la dieta (Gibbons y Tinkle 1969; Wallis et al. 1999), que aunque no fueron variables medidas en este trabajo, si se pueden asumir como parámetros biológicos relacionados.

Para Macroclemys temmnicki (Dobie 1971), Pseudemys scripta (Moll y Legler, 1971), y Kinosternon subrubrum (Iverson 1979), los folículos de mayor tamaño fueron observados en hembras postovulatórias, hecho que no se observa en los meses siguientes, ocurre que son reabsorbidos ya que no son retenidos para futuras posturas. $P$. dumerilianus posee una sola reproducción anual según los registros de este estudio, en donde se evidencia que hay reabsorción absoluta de los folículos presentes en los ovarios cuando la hembra posee huevos oviductales, coincidiendo con lo seńalado por Dobie (1971), Moll y Legler (1971). De esta forma y teniendo en cuenta que en los dos meses siguientes a la postura los ovocitos predominantes son menores a seis $\mathrm{mm}$ de diámetro y se considera, según Iverson y Moll (1997) que son inactivos, entonces la reabsorción de ellos puede entenderse como un proceso rápido que se desarrolla casi al mismo tiempo con la reabsorción de los cuerpos lúteos, no dejando opción fisiológica para un segundo y sucesivo evento reproductivo durante el ciclo anual.

Según Gemmell (1995) el papel principal del cuerpo lúteo es el mantenimiento de la gravidez o de los huevos oviductales en desarrollo para las especies de fertilización interna; Guillette et al. (1995), conceptúan que la estructura del cuerpo lúteo en quelonios es similar a la que exhiben algunos mamíferos, caracterizada por la secreción de grandes cantidades de progesterona y mínimas cantidades de esteroides sexuales, como testosterona y estradiol; Altland (1951) indica que la mayor actividad hallada para el cuerpo lúteo en Terrapene carolina coincide con la formación de membranas externas en el oviducto, sugiriendo su influencia en el proceso, lo que puede entonces reafirmar el rápido desaparecimiento del cuerpo lúteo una vez que ocurre la postura como se observó en $P$. dumerilianus. Al respecto no se coincide con la planteado por Moll y Legler (1971) referente a P. scripta, en donde la permanencia del cuerpo lúteo glandularmente activo se mantiene mientras existen huevos oviductal.

Por su parte Miller (1959) plantea que generalmente el cuerpo lúteo en reptiles ovíparos regresa poco tiempo después de la postura, coincidiendo con lo hallado por Legler (1960) para Terrapene ornata y con este estudio, y contrario a lo establecido por Webb (1961) quien asume una regresión completa a los tres meses siguientes a la postura.

La cantidad de huevos oviductales encontrados no muestra diferencia significativa entre los dos oviductos, hecho que coincide con lo registrado para Stenotherus odoratus por Dobie (1971), quien también halló igual número de huevos por oviducto, pero difiere con lo estimado por Legler (1960) para T. ornata que probablemente alterna los oviductos de acuerdo con el tamaño de los huevos por año, mostrando diferencia en el numero de huevos por oviducto.

En el presente estudio por primera vez se analiza el ciclo ovárico de $P$. dumerilianus y se evidencia por los resultados que existe una jerarquía ovárico folicular marcada, que determina la existencia de una sola postura por año, la cual ocurre sincrónicamente en el área de estudio durante la época de sequía o pulso bajo del río, igualmente se determina que la relación entre tamańo de la hembra y masa ovárica es significativa.

\section{AGRADECIMIENTOS}

Los autores agradecen a FAPEAM por la financiación del Proyecto, al INPA por la disposición de equipos, materiales y apoyo, a la Fundación Rio Negro Lodge por su apoyo logístico durante las faenas de campo, a IBAMA por las autorizaciones concedidas.

\section{BIBLIOGRAFIA CITADA}

Altland, P.D. 1951. Observations on the structure of the reproductive organs of the box turtle. Journal of Morphology, 89: 599-621.

Avendaño, I.; Muñoz, A.; Varela, N. 2002. Aproximación al conocimiento sobre la reproducción de quelonios. Portal Veterinaria, 1: 1-10.

Bernhard, R. 2001. Biologia reprodutiva de Podocnemis sextuberculata (Testudines, Pelomedusidae) na Reserva de Desenvolvimento Sustentável Mamirauá, Amazonas, Brasil. Dissertação de Mestrado, Instituto Nacional de Pesquisas da Amazônial Universidade Federal do Amazonas, Manaus, Amazonas. 62pp.

Brooks, R.J.; Shilton, C.M.; Brown, G.P.; Quinn, N.W.S. 1992. Body size, age distribution, and reproduction in a northern 
population of wood turtles (Clemmys insculpta). Canadian Journal of Zoology, 70(3): 462-469.

Clark, P.J.; Ewert, M.A.; Nelson, C.E. 2001. Physical apertures as constraints on egg size and shape in the Common Musk Turtle, Sternotherus odoratus. Functional Ecology, 15: 70-77.

Congdon, J.D.; Gibbons, J.W. 1985. Egg components and reproductive characteristics of turtles: Relationships to body size. Herpetologica, 41(2): 194-205.

Congdon, J.D.; Gibbons, J.W. 1987. Morphological constraint on egg size: a challenge to optimal egg size theory? Proceeding of the National Academy of Sciences of the USA, 84: 4145-4147.

Daza. J.M.; Páez, V.P. 2007. Morphometric variation and its effect on reproductive potential in female Colombian slider turtles (Trachemys callirostris callirostris). Herpetologica, 63(2): 125-134.

Dobie, J.L. 1971. Reproduction and growth in the alligator snapping turtle, Macroclemys temmincki (troost). Copeia, 1971: 645-658.

Etches, R.J.; Petitte, J.N. 1990. Reptilian and avian follicular hierarchies: models for the study of ovarian development. The Journal Experimental Zoology, Supplement 4: 112-122.

Félix-Silva, D. 2004. Ecologia Reprodutiva do "Cabeçudo" (Peltocephalus dumerilianus) Testudines: Pelomedusidae, no Parque Nacional do Jaú, Amazonas. Dissertação de Mestrado, Universidade Federal do Rio de Janeiro, Rio de Janeiro. 121pp.

Gemmell, R.T. 1995. A comparative study of the corpus luteum. Reproduction, Fertility and Development, 7(3): 303-312.

Gibbons, J.W.; Greene, J.L.; Patterson, K.K. 1982. Variation in Reproductive charactristics of aquatic turtles. Copeia, 1982: 776-784.

Gibbons, J.W.; Tinkle, D.W. 1969. Reproductive variation between turtle populations in a single geographic area. Ecology, 50(2): 340-341.

Girling, J.E. 2002. The reptilian oviduct: a review of structure and function and directions for future research. The Journal of Experimental Zoology, 293(2): 141-170.

Guillette, L.J.; Woodward, A.R.; You-Xiang, Q.; Cox, M.C.; Matter, J.M.; Gross, T.S. 1995. Formation and regression of the Corpus Luteumof the American alligator (Alligator mississipiensis). Journal of Morphology, 224: 97-110.

Iverson, J.B. 1979. Reproduction and growth of the mud turtle, Kinosternon subrubrum (Reptilia, Testudinata, Kinosternidae), in Arkansas. Journal of Herpetology, 13 (1): 105-111.

Iverson, J.B.; Moll, P.E. 1997. The female reproductive cycle of the Florida softshell turtle (Apalone ferax). Journal of Herpetology, 3(3): 399-409.

Iverson, J.B.; Smith, J. 1993. Reproductive ecology of the Painted Turtle (Chrysemys picta) in the Nebraska sandhills and across its range. Copeia, 1-21.

Legler, J.M. 1960. Natural history of the ornate box turtle, Terrapene ornata ornata Agassiz. University of Kansas Publications, Museum of Natural History, 11: 527-669.

Mahmoud, I.Y.; Klicka, J. 1979. Feeding, Drinking, and Excretion. p. 229-246. In. Harless. M.; Morlock, H.;(Eds) John Wileyand Sons. Turtles Perspectives and Research. New York, USA.
Medem, F. 1983. La Reproducción De La Tortuga "Cabezon" Peltocephalus tracaxa (Spix). 1924. (Testudines. Pelomedusidae) en Colombia. Lozania, Acta Zoológica Colombiana, 41: 1-12.

Miller, M.R. 1959. The endocrine basis for reproductive adaptations in reptiles. p. 449-516. In: Gorbman, A. (Ed). Comparative endocrinology. John Wiley and Sons. New York.

Mitchell, J.C. 1985. Female reproductive cycle and life history attributes in a Virginia population of stinkpot turtles, Stenotherus odoratus. Copeia, 1985(4): 941-949.

Moll, E.O.; Legler, J. 1971. The life history of a neotropical Slider Turtle, Pseudemys scripta (Schoepff) in Panamá. Bulletin of the Los Angeles Musseum of Natural History Science, 11: 1-102.

Neiff, J. J.; Iriondo, M.H.; Carignan, R.1994. Large tropical South American wetlands: an overview. p.156-165. In: Link, G. L.; Naiman, R.J. (Eds). The Ecology and Management of Aquaticterrestrial Ecotones. Proceedings, University of Washington, Seattle.

Nieuwolt-Dacanay, P.M. 1997. Reproduction in the Western Box Turtle, Terrapene ornata luteola. Copeia, 1967(4): 819-826.

Pezzuti, J.C.B. 1998. Reprodução da iaçá, Podocnemis sextuberculata (Testudines, Pelomedusidae), na Reserva de Desenvolvimento Sustentável Mamirauá. Dissertação de Mestrado, Instituto Nacional de Pesquisas da Amazônia/Universidade Federal do Amazonas, Manaus, Amazonas. 68pp.

Ramo, C. 1982. Biología del galápago Podocnemis vogli (MULLER, 1935) en hato el Frío, llanos de Apure (Venezuela). Doñana Acta Vertebrata, 9(3): 1-61.

Rowe, J.W. 1994. Egg size and shape variation within and among Nebraskan Painted Turtle (Chrysemys picta bellii) populations: Relationships to clutch and maternal body size. Copeia, 1994(4): 1034-1040.

Schōngart, J.; Piedade, M.T.; Wittmann, F.; Junk, W.J.; Worbes, M. 2005. Wood growth patterns of Macrolobium acaciifolium Benth. (Fabaceae) in Amazonian black-water and white-water floodplain forests. Oecologia, 145: 454-461.

Sioli, H. 1975. Tropical rivers as expressions for their terrestrial environments. Trends in terrestrial and aquatic research, $\mathrm{p}$. 275-288. In: Golley, F.B.; Medina, E. (Eds). Tropical Ecological Systems. Springer-Verlag, New York.

Smith, C.C.; Fretwell, S.D. 1974. The optimal balance between size and number of offspring. The American Naturalist, 108: 499-506.

Souza, R.R.; Vogt, R.C. 1994. Incubation temperature influences sex and hatchling size in the Neotropical turtle Podocnemis uniflis. Journal of Herpetology, 28(4): 453-464.

Tucker, J.K.; Janzen, F.J. 1998. Order of oviposition and egg size in the red-eared slider turtle (Trachemys scripta elegans). Canadian Journal of Zoology, 76: 3777-3380.

Valenzuela, N. 2001. Constant, shift, and natural temperature effects on sex determination in Podocnemis expansa turtles. Ecology, 82(11): 3010-3024.

Vanzolini, P.E. 1977. A brief biometrical note on the reproductive biology of some South American Podocnemis (Testudines, Pelomedusidae). Papéis Avulsos de Zoologia, São Paulo, 31(5): 79-102. 


\section{ACTA}

Vanzolini, P.E. 2003. On clutch size and hatching success of the South American turtles Podocnemis expansa (Schweigger, 1812) and P. unifilis Troschel, 1848 (Testudines, Podocnemididae). Anais da Academia Brasileira de Ciências, 75(4): 415-430.

Vanzolini, P.E.; Gomes, N. 1979. A Note on the biometry and reproduction of Podocnemis sextuberculata (Testudines, Pelomedusidae). Papéis Avulsos de Zoologia, 32(23): 277-290.

Vogt, R.C. 2001. Turtles of Rio Negro. p. 245-262 In: Chao, N.L.; Petry, P.; Prang, G.;Sonneschien, L.; Tlusty, M. (Eds). Conservation and management of ornamental fish resources of the Rio Negro Basin, Amazonia. Projeto Piaba. Universidade do Amazonas, Manaus, Brasil.

Vogt, R.C.; Cantarelli, V.C.; De Carvalho, G.A. 1994. Reproduction of the Cabeçudo, in the Biological Reserve of Río Trombetas, Pará, Brazil. Chelonian Conservation and Biology, 1(2): 145-148.
Wallis, I.R.; Henen, B.T.; Nagy, K.A. 1999. Egg size and annual egg production by female desert tortoises (Gopherus agassizii): the importance of food abundance, body size, and date of egg shelling. Journal of Herpetology, 33(3): 394-408.

Webb, R.G. 1961. Observations on life histories of turtles (genus Pseudemys and Graptemys) in Lake Texoma, Oklahoma. American Midland Naturalist, 65(10): 193-214.

Zar, J.H. 1996. Bioestatistical analysis. Third edition. Prentice-Hall, Inc. Englewood Cliff, N.J. 432 pp.

Zuffi, M.A.; Odetti, F.; Meozzi, P. 1999. Body size and clutch size in the European 1pond turtle (Emys orbicularis) from central Italy. Journal of Zoology, 247: 139-143

Recebido em 06/01/2010

Aceito em 20/01/2010 
